\title{
Dose-dense weekly paclitaxel and carboplatin compared with conventional paclitaxel and carboplatin treatment for stage II-IV ovarian cancer patients
}

\section{Zhenhua Du and Xiaolin Ma}

Department of Obstetrics and Gynecology, Sheng Jing Hospital of China Medical University, Shenyang, Liaoning 110 022, China.

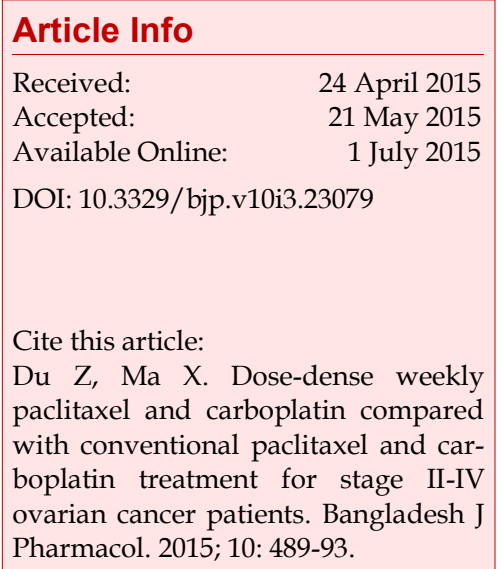

\begin{abstract}
We investigated dose-dense weekly paclitaxel and carboplatin compared with conventional paclitaxel and carboplatin treatment on stage II-IV ovarian cancer patients. Between July, 2011, and October, 2014, a total of 221 patients was randomly assigned to receive dose-dense weekly paclitaxel and carboplatin group $(n=109)$ and conventional paclitaxel and carboplatin group ( $n=112$ ), just after the sixth chemotherapy cycles, and at 12 months after randomization. Median progression-free survival (PFS) was 16.8 months (range 3.3-48+ months) of conventional paclitaxel and carboplatin group was lower than that of dose-dense weekly paclitaxel and carboplatin group 27.6 months (range 4.2-51+ months). But, these clinical responses were not statistical significance in each group. In conclusion, dose-dense weekly paclitaxel and carboplatin treatment improves survival compared with conventional paclitaxel and carboplatin treatment.
\end{abstract}

\section{Introduction}

Ovarian cancer is a serious disease which can threat women's life and health, its morbidity is highest in the disease of gynecology, and the mortality is also high, to the end-stage ovarian cancer patients, long-term survival rate is no more than 20\% (Pu et al., 2014; Xie et al., 2014). Ovarian malignant tumor is one of the three malignant tumors in female reproductive system, it has high morbidity which is secondary to the cervical cancer and endometrial carcinoma, because of the onset of the disease is hidden, difficult early diagnosis, so when the ovarian cancer is diagnosed, $70 \%$ of them belong to the end-stage (Chen et al., 2014). Postoperative relapse may occur easily. There may be abdominal and pelvic lymph node metastasis. Patients with end-stage ovarian cancer have poor prognosis, and the fatality rate is top among the gynecology malignant tumor (Robinson et al., 2008).

So far, the treatments of ovarian cancer are still mainly operation and combine with chemotherapy and radiation (Harano et al., 2014). The epithelial carcinoma is more sensitive to chemotherapy, but because of the diversity of its biological characteristics and the characteristics of easy recurrence, transfer, even get timely treatment early or late-stage ideal tumor cells reductive surgery and give first-line chemotherapy and get clinical alleviate, still $60 \%$ of the patients have recurrence and metastasis after a period of time (Numazaki et al., 2006; Silasi et al., 2006). Conventional chemothe-rapy regimens of epithelial ovarian cancer is the combine chemotherapy based on platinum, in the platinum, cisplatin and carboplatin have a similar curative effect, but the adverse reaction is different, as neurotoxicity adverse reaction of cisplatin overlap paclitaxel, but the renal toxicity, gastrointestinal reaction and neurotoxicity of carboplatin are all less than cisplatin, so the ovarian cancer treatment guidelines make by most of the gynecological cancer center and authority of the gynecological tumors, recommend paclitaxel and carbo- 
platin program as the post-surgery first-line chemotherapy of the epithelial ovarian cancer, alleviate the adverse reaction of chemotherapy to a certain extent, and improve the patient's quality of life (Chen et al., 2013; Katsumata et al., 2013; Katsumata et al., 2009). In the present study, we investigated dose-dense weekly paclitaxel and carboplatin compared with conventional paclitaxel and carboplatin treatment on stage II-IV ovarian cancer patients.

\section{Materials and Methods}

Patients and eligibility: Between July, 2011, and October, 2014, a total of 221 patients were randomly assigned to receive dose-dense weekly paclitaxel and carboplatin group ( $\mathrm{n}=109)$ and conventional paclitaxel and carboplatin group ( $n=112)$, just after the sixth chemotherapy cycles, and at 12 months after randomization (Harano et al., 2014). The specific information of the trial has been already covered. To put it in a nutshell, it was confirmed that the suitable patients were diagnosed with stage II-IV epithelial ovarian cancer, primary peritoneal cancer, or fallopian tube cancer in histological or cytological terms. Additional criteria for inclusion consisted of age of 20 years or more, an Eastern Cooperative Oncology Group performance status of 03 , and satisfactory bone marrow, renal, and hepatic function. Patients who suffered from an ovarian tumor with a low malignant potential or a synchronous or metachronous (within 5 years) malignancies other than carcinoma in situ were barred from participation. Informed consent was provided by all patients before they took part in the study. The study protocol gained approval from the institutional review boards at each and every participating center.

The protocol was coordinated by the ShengJing Hospital of China Medical University (protocol number

\section{SJCMU3013).}

Treatment plan: Patients were expected to receive paclitaxel (taxol) and carboplatin as part of either an conventional paclitaxel and carboplatin treatment (group) or dose-dense weekly paclitaxel and carboplatin treatment (group) at random. The two groups were provided with carboplatin at a dose intended to bring about an area under the plasma concentration-time curve of $6 \mathrm{mg} /$ $\mathrm{mL} / \mathrm{min}$ on the first day of a 21-day cycle. Carboplatin was dispensed as intravenous infusion during the course of 1 hour. In addition, the conventional paclitaxel and carboplatin group received paclitaxel as intravenous infusion for 3 hours at a dose of $180 \mathrm{mg} / \mathrm{m}^{2}$ on the first day. In dose-dense weekly paclitaxel and carboplatin group, paclitaxel was dispensed as intravenous infusion over 1 hour at a dose of $80 \mathrm{mg} / \mathrm{m}^{2}$ on the first, eighth and 15th day respectively. The treatments were replicated every 3 weeks for six cycles. For those patients with measurable lesions and a partial or complete response, three extra cycles of chemotherapy were provided.

Curative effect analysis: Assessment of the response was scheduled for the $56^{\text {th }}$ day. Patients who were given cisplatin at least three times a week were deemed evaluable for response. Definitions of the response were in accordance with WHO criteria; responses was supposed to be validated by two observations at an interval of at least 4 weeks. All responses were reviewed by an experienced medical member who did not participate in the study. Providing some difference existed in the response assessment between investigator and independent reviewer, the worst response was regarded as the actual response. Disease worsening was interpreted as the emergence of a new lesion and/or $>25 \%$ increase of measurable lesions. CA-125 elevation itself was not thought of as sufficient evidence for disease progression or recurrence.

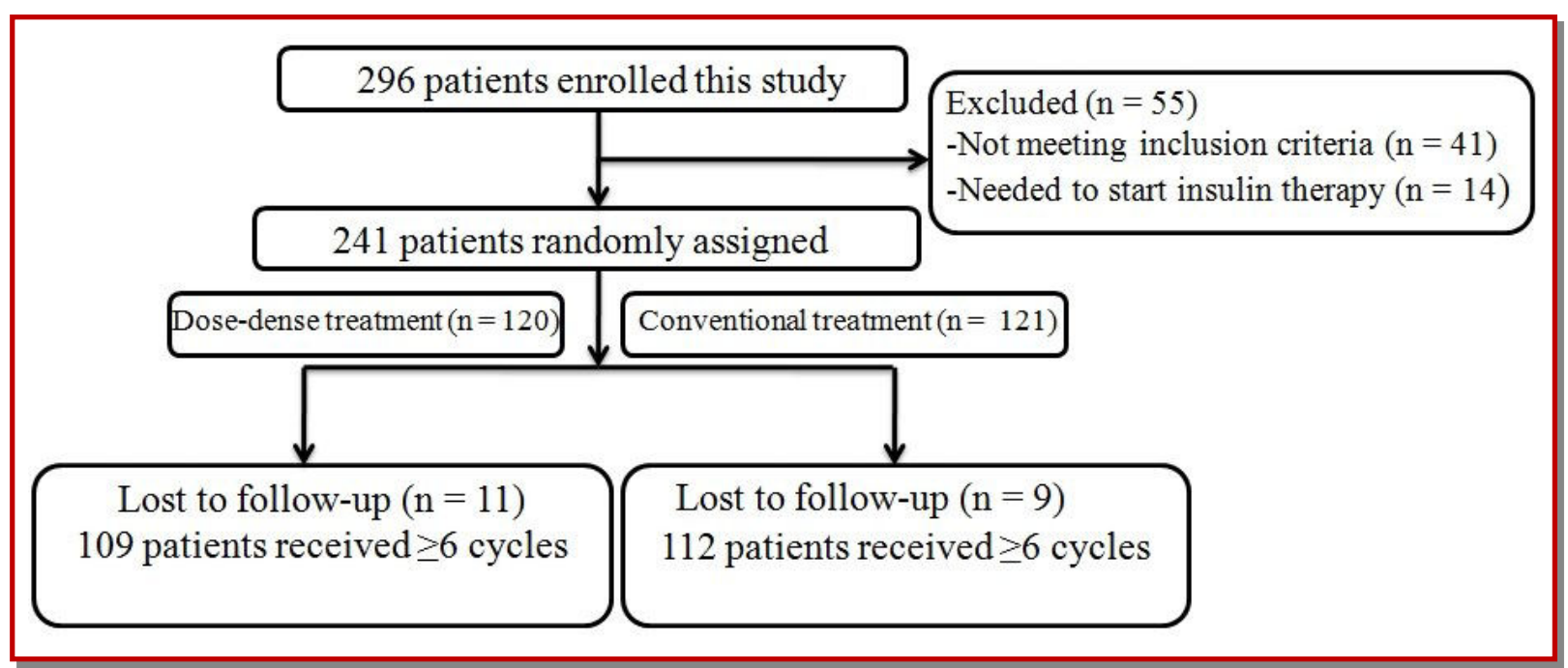

Figure 1: Trial profile 
Measurement was taken in relation to patient survival from the first day of the present treatment. The progression-free survival (PFS) probabilities were worked out by making use of the Kaplan-Meier method, with survival times for survivors determined. A non-parametric log-rank test was put to use for the purpose of testing the null hypothesis that groups to be set side by side came from the same population with regard to survival experience. NCSS v5.X (J.L. Hintze, East Kayesville, UT, USA) was taken advantage of for analysis of relevant statistics.

Statistical analysis: The SPSS 17.0 software was applied to complete data processing. All quantitative values are performed at least three times and given as mean \pm S.D. Statistical significance was analyzed by Student's t-test. Values of $p<0.05$ were considered statistically significant.

\section{Results}

Patient characteristics: Between July, 2011, and October, 2014, a total of 221 patients was randomly assigned to receive dose-dense weekly paclitaxel and carboplatin group ( $\mathrm{n}=109)$ and conventional paclitaxel and carboplatin group $(n=112)$, just after the sixth chemotherapy cycles, and at 12 months after randomization. Figure 1 showed the trial profile. Of these, 109 patients were randomized to the dose-dense weekly paclitaxel and carboplatin group and 112 were randomized to the conventional paclitaxel and carboplatin group. Baseline characteristics in our study did not differ significantly

\begin{tabular}{|c|c|c|c|}
\hline \multicolumn{4}{|c|}{ Table I } \\
\hline \multicolumn{4}{|c|}{ Patient characteristics } \\
\hline & $\begin{array}{l}\text { Dose-dense } \\
\text { treatment } \\
(\mathrm{n}=109)\end{array}$ & $\begin{array}{l}\text { Convention- } \\
\text { al treatment } \\
\quad(n=112)\end{array}$ & $\begin{array}{c}\mathrm{p} \\
\text { value }\end{array}$ \\
\hline $\begin{array}{l}\text { Age (range) in } \\
\text { year }\end{array}$ & $57(27-76)$ & $58(28-75)$ & 0.71 \\
\hline \multicolumn{4}{|l|}{ ECOG PS } \\
\hline $0-1$ & $99(90.8)$ & $101(90.2)$ & 0.56 \\
\hline 2 & $7(6.4)$ & $9(8.0)$ & \\
\hline 3 & $3(2.8)$ & $2(1.8)$ & \\
\hline \multicolumn{4}{|l|}{ FIGO stage } \\
\hline II & $20(18.3)$ & $22(19.6)$ & 0.77 \\
\hline III & $5(4.6)$ & $6(5.4)$ & \\
\hline III & $8(7.3)$ & $7(6.3)$ & \\
\hline III & $53(48.6)$ & $55(49.1)$ & \\
\hline IV & $23(21.2)$ & $22(19.6)$ & \\
\hline \multicolumn{4}{|l|}{ Histological type } \\
\hline Serous/others & $92(84.4)$ & $94(83.9)$ & 0.43 \\
\hline $\begin{array}{l}\text { Clear cell/ } \\
\text { mucinous }\end{array}$ & $17(15.6)$ & $18(16.1)$ & \\
\hline Residual disease & & & 0.56 \\
\hline$\leq 1 \mathrm{~cm}$ & 53 (48.6) & 55 (49.1) & \\
\hline$>1 \mathrm{~cm}$ & $56(51.4)$ & 57 (50.9) & \\
\hline
\end{tabular}

between the two treatment groups (Table I).

Progression-free survival: In dose-dense weekly paclitaxel and carboplatin group, median PFS was 27.6 months (range 4.2-51+ months). Median PFS was 16.8 months (range 3.3-48+ months) in conventional paclitaxel and carboplatin group and lower than that of dose-dense weekly paclitaxel and carboplatin group.

Clinical response: Table II showed that selected patients appeared complete response $(22 / 109,20.2 \%)$, partial response $(41 / 109,37.6 \%)$, stable disease (32/109, $29.4 \%)$, progressive disease $(3 / 109,2.8 \%)$ and not evaluable $(11 / 109,10.1 \%)$ in dose-dense weekly paclitaxel and carboplatin group. There 24/112 (21.4\%) patients, $43 / 112(38.5 \%)$ patients, $30 / 112(26.5 \%)$ patients, $4 / 112$ $(3.5 \%)$ patients and 11/112 (9.8\%) patients were surveyed complete response, partial response, stable disease, progressive disease and not evaluable in conventional paclitaxel and carboplatin group, respectively. These clinical responses were not statistical significance (Table II).

Adverse events: Table III showed there was statistical significance in neutropenia and anemia of ovarian cancer patients between dose-dense weekly paclitaxel and carboplatin group and conventional paclitaxel and carboplatin group. Meanwhile, thrombocytopenia, febrile neutropenia, nausea, vomiting, diarrhea, fatigue, neuropathy (motor) and neuropathy (sensory) of selected patients were observed in dose-dense weekly paclitaxel and carboplatin group and conventional paclitaxel and carboplatin group, these differences were not statistical significance.

\section{Discussion}

Ovarian cancer is one of the three most famous malignant tumor in women, mainly epithelial ovarian cancer, $80-90 \%$ of ovarian malignant tumors. Due to the ovaries deep in the pelvic cavity, concealment, it brings

\begin{tabular}{|c|c|c|c|}
\hline \multicolumn{4}{|c|}{ Table II } \\
\hline \multicolumn{4}{|c|}{ Clinical responses } \\
\hline & $\begin{array}{l}\text { Dose-dense } \\
\text { treatment } \\
(n=109)\end{array}$ & $\begin{array}{l}\text { Convention- } \\
\text { al treatment } \\
\quad(n=112)\end{array}$ & $\underset{\text { value }}{\mathrm{p}}$ \\
\hline $\begin{array}{l}\text { Complete } \\
\text { response }\end{array}$ & $22(20.2)$ & $24(21.4)$ & 0.56 \\
\hline Partial response & $41(37.6)$ & $43(38.4)$ & 0.73 \\
\hline Stable disease & $32(29.4)$ & $30(26.5)$ & 0.32 \\
\hline $\begin{array}{l}\text { Progressive } \\
\text { disease }\end{array}$ & $3(2.8)$ & $4(3.5)$ & 0.88 \\
\hline Not evaluable & $11(10.1)$ & $11(9.8)$ & 0.90 \\
\hline
\end{tabular}


the late phase (Januchowski et al., 2014). Ovarian cancer also prone to spread planting and lymph node metastasis in pelvic or abdominal cavity, although the tumor cells destroy combine the treatment of radiotherapy and chemotherapy scheme has a great improvement, but ovarian cancer recurrence rate is still high, with poor prognosis, 5 years of survival rate below $30 \%$ (Liu et al., 2014). So, in the tumor of female reproductive system, ovarian cancer has the highest fatality rate in malignant tumors. In our study, from July, 2011 to October, 2014, 221 patients were randomly assigned to the dose-dense weekly paclitaxel and carboplatin group $(n=109)$ and conventional paclitaxel and carboplatin group $(\mathrm{n}=112)$. Baseline characteristics in our study did not differ significantly between the two treatment groups.

Epithelial ovarian carcinoma is difficult to be diagnosed early, rapid progress, high malignant degree, easily abdominopelvic cavity metastasis, and prone to resistance to chemotherapy and treatment effect is poor, mortality is highest in malignant tumor (Inoue et al., 2006). In the past 30 years, the surgery treatment and radical tumor cells destroy in the early phase ovarian cancer (phase I and II) or advanced cases (phase III and phase IV) using combined chemotherapy based on cisplatin make the patients have better prognosis, but the 5-year survival rate is still around 5-30\%. Into the 1990s, as the clinical application of paclitaxel, paclitaxel, cisplatin replace cisplatin, cyclophosphamide combined chemotherapy as the post-surgery first-line treatment of ovarian cancer, prolongs the median survival time in advanced cases, to decrease the poisonousness of the PT scheme, carboplatin was used to replace cisplatin combine paclitaxel therapy, carboplatin is the second generation of platinum antitumor drugs, it's mechanism of action is the same with cisplatin, but the reaction of gastrointestinal tract and renal toxicity is lower than cisplatin (Burris et al., 2012; Kumagai et al., 2011; Mundhenke et al., 2008). Because of the advantages of patient tolerance, do not need to be hydrated, currently accepted by a lot of doctors and patients, at present the paclitaxel combine carboplatin therapy is the postsurgery first-line treatment of ovarian epithelial carcinoma at home and abroad (Harano et al., 2014). We found that dose-dense weekly paclitaxel and carboplatin increased median PFS of stage II-IV ovarian cancer patients, in comparison to that of conventional paclitaxel and carboplatin treatment. Nevertheless, the occurrence rate of neutropenia and anemia were effectively activated in dose-dense weekly paclitaxel and carboplatin treatment, comparied with those of conventional paclitaxel and carboplatin treatment.

In conclusion, these results of our study displayed that PFS of dose-dense weekly paclitaxel and carboplatin is associated with longer than a conventional regimen of paclitaxel and carboplatin in epithelial ovarian cancer.

\begin{tabular}{|lllr|}
\hline \multicolumn{4}{|c|}{ Table III } \\
\hline \multicolumn{4}{|c|}{ Adverse events } \\
\hline & $\begin{array}{c}\text { Dose-dense } \\
\text { treatment } \\
(\mathrm{n}=109)\end{array}$ & $\begin{array}{c}\text { Convention- } \\
\text { al treatment } \\
(\mathrm{n}=112)\end{array}$ & $\mathrm{p}$ value \\
\hline & $99(90.8)$ & $71(63.4)$ & $<0.01$ \\
\hline Neutropenia & $43(39.4)$ & $33(29.5)$ & 0.11 \\
Thrombocyto- & & \\
penia & $71(65.1)$ & $44(39.3)$ & $<0.01$ \\
Anemia & $10(9.2)$ & $11(9.8)$ & 0.93 \\
Febrile & & & 0.91 \\
neutropenia & $11(10.1)$ & $12(10.7)$ & 0.76 \\
Nausea & $2(1.8)$ & $3(2.7)$ & 0.97 \\
Vomiting & $4(3.7)$ & $4(3.6)$ & 0.98 \\
Diarrhoea & $5(4.6)$ & $5(4.5)$ & 0.89 \\
Fatigue & $5(4.6)$ & $6(5.4)$ & 0.82 \\
$\begin{array}{l}\text { Neuropathy } \\
\text { (motor) }\end{array}$ & $7(6.4)$ & $6(5.4)$ & \\
Neuropathy & & & \\
(sensory) & & & \\
\hline
\end{tabular}

\section{References}

Burris HA, 3rd, Dowlati A, Moss RA, Infante JR, Jones SF, Spigel DR, Levinson KT, Lindquist D, Gainer SD, Dar MM, Suttle AB, Ball HA, Tan AR. Phase I study of pazopanib in combination with paclitaxel and carboplatin given every 21 days in patients with advanced solid tumors. Mol Cancer Ther. 2012; 11: 1820-28.

Chen D, Cheng J, Yang K, Ma Y, Yang F. Retrospective analysis of chronomodulated chemotherapy versus conventional chemotherapy with paclitaxel, carboplatin, and 5-fluorouracil in patients with recurrent and/or metastatic head and neck squamous cell carcinoma. Onco Targets Ther. 2013; 6: 1507-14.

Chen K, Wei H, Ling S, Yi C. Expression and significance of transforming growth factor-beta1 in epithelial ovarian cancer and its extracellular matrix. Oncol Lett. 2014; 8: 217174 .

Harano K, Terauchi F, Katsumata N, Takahashi F, Yasuda M, Takakura S, Takano M, Yamamoto Y, Sugiyama T. Qualityof-life outcomes from a randomized phase III trial of dosedense weekly paclitaxel and carboplatin compared with conventional paclitaxel and carboplatin as a first-line treatment for stage II-IV ovarian cancer: Japanese Gynecologic Oncology Group Trial (JGOG3016). Ann Oncol. 2014; 25: 251 -57 .

Inoue A, Usui K, Ishimoto O, Matsubara N, Tanaka M, Kanbe M, Gomi K, Koinumaru S, Saijo Y, Nukiwa T. A phase II study of weekly paclitaxel combined with carboplatin for elderly patients with advanced non-small cell lung cancer. Lung Cancer. 2006; 52: 83-87.

Januchowski R, Zawierucha P, Rucinski M, Zabel M. Microarray-based detection and expression analysis of extracellular matrix proteins in drugresistant ovarian cancer 
cell lines. Oncol Rep. 2014; 32: 1981-90.

Katsumata N, Yasuda M, Isonishi S, Takahashi F, Michimae H, Kimura E, Aoki D, Jobo T, Kodama S, Terauchi F, Sugiyama $\mathrm{T}$, Ochiai K. Long-term results of dose-dense paclitaxel and carboplatin versus conventional paclitaxel and carboplatin for treatment of advanced epithelial ovarian, fallopian tube, or primary peritoneal cancer (JGOG 3016): A randomised, controlled, open-label trial. Lancet Oncol. 2013; 14: 1020-26.

Katsumata N, Yasuda M, Takahashi F, Isonishi S, Jobo T, Aoki D, Tsuda H, Sugiyama T, Kodama S, Kimura E, Ochiai K, Noda K. Dose-dense paclitaxel once a week in combination with carboplatin every 3 weeks for advanced ovarian cancer: a phase 3, open-label, randomised controlled trial. Lancet. 2009; 374: 1331-38.

Kumagai S, Sugiyama T, Shoji T, Michimae H, Katsumata N, Aoki D, Terauchi F, Jobo T, Ochiai K, Yasuda M. Does severe anemia caused by dose-dense paclitaxel-carboplatin combination therapy have an effect on the survival of patients with epithelial ovarian cancer? Retrospective analysis of the Japanese Gynecologic Oncology Group 3016 trial. Int J Gynecol Cancer. 2011; 21: 1585-91.

Liu X, Gao Y, Lu Y, Zhang J, Li L, Yin F. Down-regulation of NEK11 is associated with drug resistance in ovarian cancer. Int J Oncol. 2014; 45: 1266-74.

Mundhenke C, Weigel MT, Sturner KH, Roesel F, MeinholdHeerlein I, Bauerschlag DO, Schem C, Hilpert F, Jonat W,
Maass N. Novel treatment of ovarian cancer cell lines with imatinib mesylate combined with paclitaxel and carboplatin leads to receptor-mediated antiproliferative effects. J Cancer Res Clin Oncol. 2008; 134: 1397-405.

Numazaki R, Miyagi E, Onose R, Nakazawa T, Sugiura K, Asukai K, Nakayama H, Miyamatsu A, Okamoto N, Hirahara F. Historical control study of paclitaxel-carboplatin (TJ) versus conventional platinum-based chemotherapy (CAP) for epithelial ovarian cancer. Int J Clin Oncol. 2006; 11: 221-28.

Pu Z, Yuan X, Zhang X, Chen Q, Xie H. Meta-analysis on the association between CYP2D6*10 gene polymorphism and disease free survival of breast cancer patients receiving tamoxifen treatment in Asia. Bangladesh J Pharmacol. 2014; 9: 652-62.

Robinson WR, Davis N, Rogers AS. Paclitaxel maintenance chemotherapy following intraperitoneal chemotherapy for ovarian cancer. Int J Gynecol Cancer. 2008; 18: 891-95.

Silasi DA, Alvero AB, Illuzzi J, Kelly M, Chen R, Fu HH, Schwartz P, Rutherford T, Azodi M, Mor G. MyD88 predicts chemoresistance to paclitaxel in epithelial ovarian cancer. Yale J Biol Med. 2006; 79: 153-63.

Xie Y, Peng Z, Shi M, Ji M, Guo H, Shi H. Metformin combined with p38 MAPK inhibitor improves cisplatin sensitivity in cisplatin resistant ovarian cancer. Mol Med Rep. 2014; 10: 2346-50.

\section{Submit your next manuscript to Bangladesh Journal of Pharmacology and take full advantage of: \\ - Easy online submission \\ - Thorough peer review \\ - Available online immediately after acceptance \\ - Open access \\ - No publication charge}

